\title{
Detection of Essential Oils Adulteration: A Quick Overview and Current Challenges
}

\author{
Paul Vargas Jentzsch*1, Luis Ramos Guerrero ${ }^{2}$ and Valerian Ciobotă ${ }^{3}$ \\ ${ }^{1}$ Departamento de Ciencias Nucleares, Escuela Politécnica Nacional, Quito, Ecuador \\ ${ }^{2}$ Centro de Investigación de Alimentos CIAL, Universidad UTE, Ecuador \\ ${ }^{3}$ Rigaku Analytical Devices, Inc., Wilmington, USA
}

*Corresponding author: Paul Vargas Jentzsch, Departamento de Ciencias Nucleares, Facultad de Ingeniería Química y Agroindustria, Escuela Politécnica Nacional, Quito, Ecuador.

To Cite This Article: Paul Vargas Jentzsch. Detection of Essential Oils Adulteration: A Quick Overview and Current Challenges. Am J Biomed Sci \& Res. 2019 - 4(1). AJBSR.MS.ID.000746. DOI: 10.34297/AJBSR.2019.04.000746

Received: July 01, 2019 | Published: July 15, 2019

\section{Opinion}

Essential oils are highly appreciated raw materials due to a variety of their intrinsic characteristics and properties. They are complex mixtures of volatile compounds produced by plants as secondary metabolites; communicational and defence roles were attributed to constituents of essential oils [1]. Over the years, many researchers have reported a great variety of properties of essential oils including antimicrobial and antioxidant activities, as well as, some other effects of biological interest that could be used in human and veterinary medicine [2]. Therefore, not only wellknown essential oils are more demanded in the market, but also enormous efforts are focused on the extraction and characterization of alternative sources of these products. From an economic point of view, this fact can significate the opening of opportunities for developing countries in South Asia, Africa, and Latin America since many of them have great biodiversity within their territories and essential oils prices tend to increase year by year. Moreover, the technologies for the extraction of essential oils (steam distillation, hydro distillation, cold expression, and supercritical fluid extraction) are well known and available at reasonable costs.

Only considering the high demand and the highly appreciated properties of some essential oils, for example, those extracted from lavender or frankincense, there is no wonder that counterfeit and adulterated essential oils can be offered in the market. The formulation of medicinal products with counterfeit and adulterated essential oils could be very dangerous and put in serious risk the health of consumers. As an example, clove essential oil is used in dental care, in fact, the characteristic odour of a dentistry office is due to eugenol, the main component of this essential oil. In earlier work, we reported the occurrence in the market of adulterated clove essential oil, and the adulterant was benzyl alcohol [3]. It is not difficult to imagine the adverse effects on the health of a person treated with a product based on clove essential oil with benzyl alcohol as adulterant. It is out of question that strict controls must be carried out, not only by governmental institution but also by companies working with essential oils as raw materials. One of the aims of using essential oils instead of synthetic substances in cosmetic and pharma products (among others) is to obtain natural products, which have fewer secondary effects and high acceptance by the customers. Clearly, the adulteration by the illegal addition of cheaper substances to essential oils modifies the quality of the final product and goes against this aim.

Currently, the technique considered the gold standard for the quality control of essential oils is gas chromatography (GC), ideally, coupled to mass spectrometry (MS). By methods based on this technique, the detection of volatile compounds present in essential oils is possible and even the confirmation of the identity of such compounds can easily be achieved. To deal with the adulteration based on the addition of synthetic versions of compounds present in the essential oils, isotope-ratio mass spectrometry and chiral GC analysis have demonstrated great efficiency [4,5]. In cases in which the adulteration with a non-volatile substance is suspected, the use of liquid chromatography (i.e., high performance liquid chromatography, HPLC) could be useful [6]. However, this technique is not routinely used for quality control in industries and methods based on GC are still predominant with the consequent limitations.

The adulteration of essential oils by the addition of nonvolatile substances is a very concerning issue that seems to be underestimated. In an earlier study on clove essential oils [3], we found that a commercial sample was adulterated with a vegetable oil. While (in this work) it was relatively easy to detect the adulterant benzyl alcohol by GC/MS/MS, the vegetable oil was not detected by mean of this technique. Only volatile and semi volatile compounds can be detected with a GC system because this technique requires 
the vaporization of analyte for its introduction into the column. Basically, in a GC analysis, any non-volatile substance (in this case, the adulterant) is retained in the injection port and the chromatogram shows peaks only of the volatile constituents, i.e., the expected constituents of the essential oil. Changes in the areas of the peaks could be a clue of adulteration, however, such changes could also be attributed to regional and seasonal variations. Earlier studies have demonstrated that the composition of an essential oil shows certain variations due to the location where the plants were grown and the season [7]. Therefore, the detection of adulterated essential oils by GC methods has serious limitations when it comes to deal with non-volatile adulterants. In such cases, the application of spectroscopic techniques may be more appropriate. In fact, the application of Raman spectroscopy to the quality control of essential oils has some advantages; the Raman spectra of essential oils consist in sharp bands that can easily be assigned to specific compounds [8] and the presence of adulterants can be noticeable by the occurrence of new bands and/or changes in the shape of the bands. Even slight changes in the spectra may be observable by data processing with chemometric methods (e.g., Principal Components Analysis, PCA). Moreover, since portable and handheld Raman spectrometers are already commercially available, the possibility of performing in situ measurements provides more versatility to quality control activities.

As mentioned before, essential oils have already important applications in human and veterinary medicine, and clearly, they will gain in importance in years ahead. Many authors have considered the possible substitution of some antibiotics by essential oils or individual components they contain $[9,10]$. This substitution may start in activities related to animal production and then, perhaps, also find its way to the treatment of bacterial infections in humans. The emergence of multidrug-resistant bacteria forces not only to promote the diversification of antimicrobial substances used in veterinary and human medicine, but also the substitution of some generations of antibiotics that should be kept as the last resource against "superbugs". Only by ensuring the quality of essential oils by mean of the most appropriate analytical strategies would be possible to obtain the best benefits of these products.

\section{References}

1. R Pavela, G Benelli (2016) Essential oils as Ecofriendly biopesticides? Challenges and constraints. Trends Plant Sci 21(12): 1000-1007.

2. AE Edris (2007) Pharmaceutical and therapeutic potentials of essential oils and their individual volatile constituents: a review. Phytother Res 21(4): 308-323.

3. P Vargas Jentzsch, F Gualpa, LA Ramos, V Ciobotă (2017) Adulteration of clove essential oil: Detection using a handheld Raman spectrometer. Flavour Fragrance J 33(2): 184-190.

4. L Schipilliti, P Dugo, I Bonaccorsi, L Mondello (2012) Authenticity control on lemon essential oils employing Gas ChromatographyCombustion-Isotope Ratio Mass Spectrometry (GC-C-IRMS). Food Chem 131(4): 1523-1530.

5. S Paraschos, P Magiatis, E Gikas, I Smyrnioudis, AL Skaltsounis, et al. (2016) Quality profile determination of Chios mastic gum essential oil and detection of adulteration in mastic oil products with the application of chiral and non-chiral GC-MS analysis. Fitoterapia 114: 12-17.

6. H Fan, Q Wu, JE Simon, SN Lou, CT Ho, et al. (2015) Authenticity analysis of citrus essential oils by HPLC-UV-MS on oxygenated heterocyclic components. J Food Drug Anal 23(1): 30-39.

7. LC Soro, S Munier, Y Pelissier, L Grosmaire, R Yada, et al. (2016) Influence of geography, seasons and pedology on chemical composition and antiinflammatory activities of essential oils from Lippia multiflora Mold leaves. J Ethnopharmacol 194: 587-594.

8. P Vargas Jentzsch, LA Ramos, V Ciobotă (2015) Handheld Raman Spectroscopy for the Distinction of Essential Oils Used in the Cosmetics Industry. Cosmetics 2(2): 162-176.

9. D Savoia (2012) Plant-derived antimicrobial compounds: alternatives to antibiotics. Future Microbiol 7(8): 979-990.

10. S Chouhan, K Sharma, S Guleria (2017) Antimicrobial Activity of Some Essential Oils-Present Status and Future Perspectives. Medicines (Basel) 4(3): 58. 